\title{
5G como oportunidad para la ruptura del duopolio en el mercado móvil
}

\section{$5 \mathrm{G}$ as an opportunity for the rupture of the duopoly in the mobile market}

\author{
Francisco Vacas-Aguilar
}

Cómo citar este artículo:

Vacas-Aguilar, Francisco (2019). "5G como oportunidad para la ruptura del duopolio en el mercado móvil". El profesional de la información, v. 28, n. 6, e280623.

https://doi.org/10.3145/epi.2019.nov.23

Artículo recibido el 15-01-2019 Aceptación definitiva: 27-06-2019

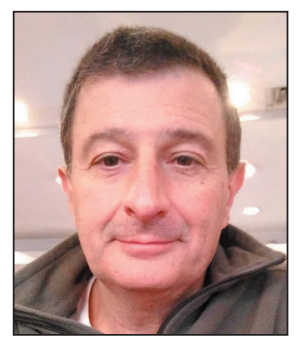

Francisco Vacas-Aguilar

https://orcid.org/0000-0003-4457-3057

Universidad Rey Juan Carlos

Facultad de Ciencias de la Comunicación

Paseo de los Artilleros, s/n.

28032 Vicálvaro (Madrid), España

pvacas2006@gmail.com

\section{Resumen}

La transformación de las redes móviles en el principal soporte de acceso a internet a principios de este siglo XXI, convirtieron a Apple y Alphabet en las empresas más lucrativas de un mercado donde hasta entonces no estaban presentes. La reinterpretación del modelo de plataforma como mercados multilaterales donde se tiende a incrementar el valor de los proveedores de servicios renunciando en gran medida al control sobre la oferta relegaron a las operadoras de telecomunicaciones al papel de proveedores de acceso en un mercado construido sobre sus propias redes. La evolución hacia el $5 \mathrm{G}$ podría revertir esta tendencia a la concentración al abrir nuevos mercados para las operadoras en el sector industrial dentro de la internet de las cosas, pero también profundizando en la personalización de la oferta al usuario. Este articulo analiza los principales hitos en la evolución de las redes móviles, las estrategias empleadas por Apple y Alphabet para posicionarse como líderes en los nichos más rentables, las características básicas del $5 \mathrm{G}$ y por qué este sistema puede suponer una oportunidad para las operadoras para superar el actual duopolio.

\section{Palabras clave}

Telefonía móvil; Tecnología; Tecnologías de la información y la comunicación; TIC; Empresas tecnológicas; Internet; Dispositivos móviles; 5G; Internet de las cosas; Mercado móvil; Apple; Alphabet; Google; Android.

\begin{abstract}
Mobile networks transformation into the main support for Internet access since last decade made Apple and Alphabet the most lucrative companies in a market where they were not present until then. The reinterpretation of platform model as multilateral markets where the value of service providers tends to increase, largely relinquishing control over supply, relegated telecommunications operators to access providers role in a market built on their own networks. The evolution towards the $5 \mathrm{G}$ could reverse this tendency to concentration by opening new markets for operators in the industrial sector within the Internet of Things (IOT), but also deepening the personalization of the offer to the user. This article analyzes the main milestones in the evolution of mobile networks, the strategies employed by Apple and Alphabet to position themselves as leaders in the most profitable niches, the basic characteristics of $5 \mathrm{G}$ and why this system can be an opportunity for operators to overcome the current duopoly.
\end{abstract}

\section{Keywords}

Mobile telephony; Technology; Information and communication technologies; ICT; Technology companies; Internet; Mobile devices; 5G; Internet of Things; loT; Mobile market; Apple; Alphabet; Google; Android. 


\section{Introducción. Estado de la cuestión}

La transformación de una red de telecomunicaciones inicialmente dirigida al servicio telefónico básico y de dimensión nacional y regional en una red multimedia global de acceso a internet

"ha constituido la mayor innovación de las dos últimas décadas en lo que respecta al intercambio de información entre personas" (Ballon, 2009, p. 15).

Aunque este escenario fue parte de una estrategia diseñada por gobiernos y operadoras nacionales para preservar un cierto status quo en el mercado de las telecomunicaciones que protegiera a las incumbentes, su posterior desarrollo demostró ser mucho más imprevisible debido a una conjugación de factores como son el carácter abierto de internet (Varian, 2003; Zittrain, 2008), las oportunidades para la disrupción que abrieron los nuevos mercados digitales (Christensen, 1997), la convergencia que permitió la entrada de nuevos actores del mercado tecnológico (Forge et al., 2018; Curwen; Whalley, 2004) y la "plataformización" (Ballon, 2009) como modelo de referencia de toda el sector TIC (Bresnahan; Greenstein, 2014).

La importancia que adquirió en poco tiempo el sistema operativo (SO) a la hora de adquirir un dispositivo de acceso y la popularización del mercado de las aplicaciones, a partir de la App Store de Apple como un nuevo modelo de distribución de contenidos y oferta de servicios, propició un desplazamiento de la parte más lucrativa del mercado de las redes al software alojado sobre estas y el hardware o dispositivo de acceso (Kenney; Bryan, 2011).

El giro radical de Apple en un decenio (2007-2017) de empresa de computación a fabricante de móviles y el desarrollo paralelo de

"un modelo de negocio diseñado para llevar al consumidor a su ecosistema y mantenerlo allí" (Montgomerie; Roscoe, 2013),

supuso el mayor punto de inflexión en este mercado ya que desplazó el epicentro de la innovación de los operadores a los servicios sobre la plataforma.

Con todo, la mayor ventaja estratégica lograda por Apple fue convencer a los usuarios de que el valor añadido estaba en el dispositivo y los contenidos para personalizarlo y no en las redes, lo que suponía relegar a las operadoras a la categoría de commodities sin otra función aparente que la de proveedor neutral del acceso, acelerando una tendencia largamente observada en el mercado de las telecomunicaciones desde principios del siglo XXI (Holmes, 2008).

El resultado es que el liderazgo por ingresos de esta internet sobre redes móviles recae en empresas como Apple, Alphabet (propietaria de Google), Facebook y Samsung, ninguna de ellas originariamente ligada al mercado de la telefonía móvil, lo que dibuja un mercado con un alto grado de concentración que como tal limita los incentivos para la entrada de nuevos actores y, sobre todo, mayores inversiones de las operadoras en un sector que supone el $4,6 \%$ del PIB mundial (GSMA, 2019).

Dentro de esta tendencia del mercado mundial el caso de Europa es altamente significativo ya que ni su liderazgo en el desarrollo e implantación de las primeras redes digitales de telefonía móvil (Gentzoglanis; Henten, 2010), ni el de Nokia como primer fabricante mundial de móviles durante 15 años (Doz; Wilson, 2018) sirvieron de base para que Europa creara un mercado innovador sobre la nueva internet móvil con productos y servicios exportables al resto del mundo, en gran medida debido a la contradicción que siempre supuso

"desear los beneficios de una economía basada en el conocimiento sin la destrucción creativa que lleva aparejada" (Atkinson; Ezell, 2012).

Las ventajas competitivas que las empresas europeas crearon en la primera fase de las redes móviles digitales (2G) se perdieron en la transición hacia los nuevos sistemas de banda ancha (3G) un factor que se repite en todo periodo de transición tecnológica y que obliga a una reorganización empresarial para responder a las nuevas demandas (Asimakopoulos; Whalley, 2017).

A continuación se analizan los hitos más importantes del mercado de la telefonía móvil durante esta etapa, el cambio de estrategia de Apple y Alphabet que les permitió convertirse en corporaciones líderes en el mercado de la internet móvil sin poseer red propia, las características principales del 5G, para concluir analizando como podría afectar el actual duopolio de las plataformas de Apple y Alphabet al desarrollo del 5G, identificando las oportunidades que tienen las operadoras a partir de ahora.

\section{Un relevo histórico: las líneas móviles superan a las fijas}

En 2002 se produjo uno de los hitos más importantes de la historia de la telefonía móvil en particular y las telecomunicaciones en general, ese año el número de líneas móviles activas superó por primera vez al de teléfonos fijos instalados (gráfico 1). 
Desde finales del siglo XIX hasta ese año 2002, la telefonía sobre redes fijas constituyó el negocio principal del sector, de modo que hablar de telefonía era hablar de teléfonos fijos para el servicio universal básico, tanto en el mercado corporativo como en el residencial.

A pesar de que la historia del teléfono fijo forma parte de la evolución tecnológica del siglo XX, desde los primeros aparatos comercializados en EUA por Bell en 1890 hasta su sustitución en el 2002 por la telefonía móvil como principal medio de comunicación, la telefonía fija había alcanzado solamente al 17\% de la humanidad (International Telecommunications Union, 2017).

Este dato permite entender por qué el servicio telefónico a lo largo de su historia fue en realidad una excepción tecnológica para la mayoría, ya que en 100 años sólo una quinta parte de la población mundial pudo disfrutar de teléfono en sus hogares (International Telecommunications Union, 2017).

Sin embargo, en apenas 24 años (1992-2016) el número de líneas móviles activas superó al de habitantes del planeta y un año más tarde, el $68 \%$ de la población mundial era ya dueña de un dispositivo de telefonía móvil (International Telecommunications Union, 2017).

Un mundo donde la mayoría de sus habitantes es usuario de telefonía (móvil) es algo inédito en la historia de la tecnología y la comunicación. Sin embargo, el acceso mayoritario a esta tecnología no significa que todos accedan ni a los mismos servicios, ni con la misma calidad, ni al mismo coste, factor este último determinante (GSMA, 2018).

La sustitución de la telefonía fija por sistemas móviles de banda ancha ha tenido implicaciones mucho mayores que un cambio en el sistema de transmisión. La primera es que la mayor parte del tráfico de datos en internet pasó de las redes fijas a los dispositivos móviles y redes inalámbricas (Cisco, 2017), lo que supuso una completa redefinición de la oferta de servicios en internet (Issarny et al., 2011; Feijóo et al., 2009).

La segunda, la transformación de los dispositivos de terminales simplemente para hablar y mandar mensajería de texto a computadoras multifuncionales (smartphones) y su difusión global implicó a su vez dos cambios transcendentales:

- el cambio en la consideración de los smartphones de tecnología fundamentalmente empresarial y profesional y su introducción masiva en el mercado de consumo, propiciada por la aparición del primer iPhone de Apple (Sarwar; Soomro, 2013).

- un relevo en el servicio básico con una disminución de las llamadas de voz y un incremento exponencial del consumo de datos, algo que alteró la histórica estructura de ingresos de las operadoras móviles en todo el mundo (Mölleryd et al., 2010).

La tercera, y asumiendo estos cambios en la naturaleza del mercado, la prioridad de los gobiernos ha sido garantizar unas velocidades mínimas de acceso a los servicios básicos de la Red a precios asequibles en cada mercado nacional (Comisión Europea, 2013; Federal Communications Commission, 2018), de modo que el acceso universal a internet dejara de ser un paradigma y se convirtiera en una realidad. Este objetivo no obstante está todavía lejos de lograrse a pesar de los evidentes progresos en la última década que situó esta brecha digital en el $49 \%$ de la población mundial sin acceso a internet (International Telecommunications Union, 2018a).

Con esta exposición de tendencias a priori se podría concluir que es en la posesión y gestión de las redes móviles donde se encuentra la clave del desarrollo de internet y de los negocios ligados a ésta. Sin embargo, el análisis de los ingresos de los principales agentes de este sector demuestra que en realidad la parte más lucrativa de la cadena de valor se encuentra en la venta de dispositivos de acceso y en las plataformas de servicios alojadas en las redes. La paradoja por tanto es que a pesar del enorme esfuerzo inversor realizado por las operadoras a comienzos de este siglo para comprar las licencias nacionales $3 G$ (Kemplerer, 2002), la parte más rentable del negocio se trasladó en poco tiempo a los extremos de la cadena de valor.

Este hecho además condiciona la futura estrategia de las operadoras de cara al 5G, ya que un mayor tráfico de datos junto a mejores y más sofisticados servicios demandan mayor capacidad de las redes, sin que se sepa con certeza si realmente es rentable la inversión hacia la siguiente generación de redes $5 \mathrm{G}$ con los actuales modelos de negocio. 


\section{Transformación del terminal: de la telefonía al acceso a internet}

La aparición de la mensajería de texto (SMS) como primer servicio universal de trasmisión de datos entre usuarios no corporativos fue uno de las "sucesos inesperados" de la implantación de las redes de segunda generación (2G) en Europa a mediados de los años 90 (Corrocher, 2013).

Por primera vez las operadoras se vieron obligadas a discriminar sus ingresos entre voz y datos, aunque los primeros siguieran considerados como principal servicio, algo que influyó además en la forma de los terminales de usuario que dejaron de ser diseñados como un teléfono que servía únicamente para hablar.

Simultáneamente la implantación de las redes 36 en todo el mundo dio paso a un mercado bien diferente al anterior. En Europa el sistema UMTS (universal mobile telecommunications service) significó la entrada de las operadoras móviles en el mercado de los proveedores de servicios de internet (ISP) en abierta competencia con las telefónicas convencionales y los operadores de cable.

Sin embargo, este cambio en la naturaleza de las redes móviles hacia una mayor capacidad no fue la única causa de la transformación de los dispositivos móviles de básicos a smartphones. La popularización del segmento smartphone en 1997 se produjo en plena era 2G, cuando las redes GSM/GPRS (global system for mobile communications/general packet radio service) apenas permitían acceder a unos servicios muy básicos de internet con unos costes que actuaban de barrera de entrada para la mayoría.

Durante esta etapa la mayor parte de los fabricantes de móviles y operadoras comenzaron a ofrecer smartphones para el usuario corporativo, pensando más en un sustituto de la por entonces exitosa agenda electrónica (PDA, personal digital assistant) que en una computadora de mano para acceso pleno a internet.

La actual entronización del smartphone como símbolo universal del acceso a internet y sustituto de las computadoras portátiles (notebook), no se debe directamente a la evolución de las redes sino a la aparición en 2007 del primer móvil de Apple, una empresa del mercado de la computación que durante 30 años se dedicó casi exclusivamente a la venta de computadoras de escritorio.

Se puede afirmar por tanto que la transformación radical del segmento smart de la telefonía móvil se debe más a su iPhonización, entendida como la adopción universal del iPhone como modelo de referencia, que a la evolución de los sistemas móviles de banda ancha.

A partir del iPhone todos los teléfonos de gama alta de sus competidores se vieron literalmente obligados a parecerse al dispositivo de Apple, y en muchos casos fueron tecnológicamente superiores. La eliminación del teclado físico y la transformación completa del teléfono en una pantalla táctil desde la cual acceder a todas las aplicaciones y servicios, cambió para siempre la naturaleza y función de los móviles desde dispositivos para hablar a computadoras portátiles multifunción.

Con el nuevo diseño de los smartphones las llamadas de voz pasaron de uso prioritario a complementario, ya que la forma del dispositivo invitaba más al usuario a tocar y acceder a las aplicaciones que a llevárselo al oído. La respuesta de los fabricantes ante esta tendencia fue aumentar progresivamente el diámetro de pantalla de los nuevos equipos, llegando en muchos casos al límite de lo que se puede considerar portabilidad en un dispositivo (Kim; Sundar, 2014) y difuminando las diferencias con categorías superiores como las tablets.

La rápida sustitución de los teléfonos básicos por smartphones primero en EUA y posteriormente en el resto del mundo, junto a la aparición de ofertas de acceso en tarifa plana, apuntalaron definitivamente la transformación del histórico mercado de la telefonía vocal de las operadoras en otro basado en el tráfico de datos.

Sin embargo, este último escenario demostró ser también transitorio para las operadoras, ya que tanto los servicios IP (propios de internet) como las aplicaciones de mensajería, comenzaron a canibalizar no sólo el declinante mercado de las llamadas, sino también a cuestionar estrategias como las ofertas en tarifa plana para ganar clientes (Andra, 2017).

\section{Apple: de los Mac al iPhone}

La entrada de Apple en el mercado de los dispositivos móviles constituye uno de los factores más desestabilizadores, que afectó tanto al sector tecnológico como al de las telecomunicaciones, y su análisis ayuda a comprender el porqué y el cómo de la evolución del mercado en su conjunto durante este crucial periodo.

Hasta 2001 no se pudo considerar a Apple una empresa del mercado de la movilidad. Durante sus primeros 25 años de vida Apple fue un fabricante puro de dispositivos informáticos con ventas limitadas pero dirigidas a un nicho de clientes muy fiel a una cierta filosofía Apple basada en la calidad de sus componentes y el diseño del producto (Johnson et al., 2012). Ese 2001 Apple lanzó al mercado el iPod, un reproductor musical de archivos mp3 con memoria flash, que se convirtió en el catalizador del cambio para una industria musical sumida en

"una crisis por la masiva difusión de tecnologías digitales para la desintermediación de música” (Rogers, 2013, p. 129). 
Pero la intuición más importante de Apple no fue el propio aparato (de hecho había una gran variedad disponible en el mercado), sino la apertura simultánea de una tienda online de venta de canciones a precio único (0,99 dólares) que serviría de proveedor directo de contenidos para los usuarios del iPod. El iPod creó un estándar en el mercado del consumo musical, como lo hizo Sony dos décadas antes con el reproductor de casetes Walkman. A partir de entonces el negocio de la venta de música se desmaterializó casi del todo (se pasó de los CDs al archivo digital) y en consecuencia, tanto empresas discográficas como tiendas físicas disminuyeron su importancia en la cadena de valor del sector.

El propio director del sello Warner Music reconocía que:

“Apple inventó el mercado de la música digital, realmente creó la convergencia de música y tecnología y mostró a todos cómo era la economía conectada del contenido" (Nash, citado por Ingraham, 2013).

Las lecciones aprendidas durante los primeros seis años de vida del modelo iPod/iTunes llevaron a Apple a lanzar su primer teléfono móvil. En 2007 apareció el iPhone, un teléfono móvil con un diseño innovador, pero con una limitada capacidad tecnológica con respecto a los dispositivos que por entonces ofrecían los fabricantes puros como Nokia o los keitai de la operadora japonesa DoCoMo.

Uno de los mayores méritos de Apple con el iPhone fue convencer a los nuevos usuarios que éste no sólo era un smartphone, sino el prototipo de la nueva computación móvil y realmente portátil. Copiando el mismo modelo empleado en el iPod con iTunes, Apple presentó un año después la tienda de aplicaciones App Store que no solo creó un mercado global para desarrolladores de aplicaciones, sino que reinventó la forma de acceso a internet encarnada hasta entonces por la Web.

La App Store de Apple se convirtió en el gran mercado de la distribución de contenidos en internet, algo que habían intentado anteriormente sin éxito grandes grupos del mercado del entretenimiento como Time-Warner (Case, 2016) convirtiéndola en un

"mercado con mayores ingresos que sectores tradicionales como la taquilla cinematográfica o la industria musical" (Dediu, 2018).

El éxito de la tienda de aplicaciones de Apple se debe a una combinación de factores, entre los cuales está la selección de contenidos, la fijación de un precio único, la no segmentación geográfica de la oferta, la voluntad de los usuarios del iPhone de personalizar su dispositivo, algo hasta entonces reservado exclusivamente a las operadoras, pero sobre todo la posibilidad de

“poner en contacto directo a desarrolladores de software con consumidores" (Naeem, 2016, p. 44).

La táctica de Apple de crear una tienda de aplicaciones que sirviera de apoyo a la venta del dispositivo de acceso, pero sobre todo

"el férreo control de ésta sobre su plataforma multicanal con múltiples puntos de venta para sus dispositivos de hardware pero uno solo para sus contenidos" (Montgomery; Roscoe, 2013, p. 292)

no sólo supuso un modelo de éxito, sino que se convirtió en la fórmula paradigmática del mercado de la telefonía móvil y un pilar básico de cualquier estrategia en internet.

Apple logró desplazar el epicentro del negocio de las redes al terminal y los contenidos, consiguiendo que el iPhone se percibiera como

"una parte del paradigma de la nueva computación móvil que seguía la trayectoria del PC y del portátil" (Dediu, 2010).

La estrategia de Apple con el iPhone influyó de este modo en el mercado de las operadoras de telefonía móvil, obligándolas indirectamente a incrementar la capacidad de sus redes, acelerando sus ritmos de inversión e iniciando una estrategia de fidelización de sus propios clientes mediante la subvención de los terminales de acceso como el propio iPhone.

El efecto halo (Thorndike, 1920) generado por el iPhone y la amplia oferta de contenidos de la App Store volvieron a influir positivamente en 2010 cuando Apple comercializó su primera tableta iPad, otro dispositivo clave para el mercado de la movilidad.

Con todo, el mayor y por el momento más duradero efecto que tuvo la irrupción de Apple en el mercado de la movilidad con el iPhone fue el desplazamiento del centro de la innovación mundial en este sector desde Europa y Japón a EUA, que hasta entonces estaba más atrasado (Laugesen; Yuan, 2010).

Con una Apple convertida no sólo en la empresa más rentable del mercado de la movilidad (gráfico 2) sino en una de las mayores en capitalización bursátil, se alteró el ranking histórico en el mercado de las telecomunicaciones, ya que por primera vez un fabricante de dispositivos tenía una dimensión económica mayor que las operadoras de redes. 
La capacidad financiera de Apple derivada de los ingresos del iPhone durante más de 10 años es de tal magnitud que puede desequilibrar cualquier sector donde desee entrar, lo que incluye el mercado de la producción de películas y series para su propia plataforma, un sector que a pesar del reciente auge del consumo de vídeo en streaming es táctico pero "no necesariamente estratégico" para Apple (Evans, 2017).

El análisis de la trayectoria de Apple durante los últimos 10 años muestra como ha creado un sistema cerrado integrado por dispositivos, mercado de aplicaciones y un sistema operativo (iOS) que actúa como gestor de la plataforma, que representa no solamente un modelo de referencia para la industria tecnológica sino para todas las empresas del entretenimiento que han visto como las antiguas fronteras entre mercados se desvanecían.

\section{Alphabet y la estrategia Android}

Alphabet es un conglomerado de empresas que representa con precisión la propia evolución del mercado de internet en los últimos 20 años, sinónimo de disrupción (Christensen, 1997), innovación y objetivos ambiciosos (Collins, 1999), que ha hecho posible tanto la creación de nuevos mercados muy lucrativos -como la publicidad en buscadores-, como su irrupción en mercados maduros (videojuegos, infraestructura de red) o en expansión (inteligencia artificial).

El principal negocio de Alphabet es Google (Alphabet, 2018) que a lo largo de sus 20 años de existencia pasó de ser un buscador de páginas web (search engine) a convertirse en una plataforma de servicios desplegables a voluntad del usuario, siguiendo una bien meditada estrategia basada en el "modelo de la navaja suiza" (Heinemann; Gaiser, 2014, p. 121). El monopolio global de facto que Google ha logrado en el mercado de las búsquedas de webs en internet (gráfico 3), le condujo al mercado de la movilidad.

La evidencia de que las redes inalámbricas pasarían a ser en poco tiempo el soporte fundamental de la nueva internet obligó a Google a adaptar toda su oferta de servicios a los nuevos dispositivos de acceso, especialmente a los smartphones.

Pero el nuevo mercado de la internet móvil "caracterizado por ciclos de vida de producto mucho más cortos" (Schmidt; Rosenberg, 2014) no sólo llevó a Google a adoptar cambios tecnológicos y de diseño, sino que obligó a adoptar estrategias diferentes, ya que surgieron nuevos competidores, se alteró el ranking de los mercados geográficos y sobre todo, cambiaron las demandas de los usuarios.

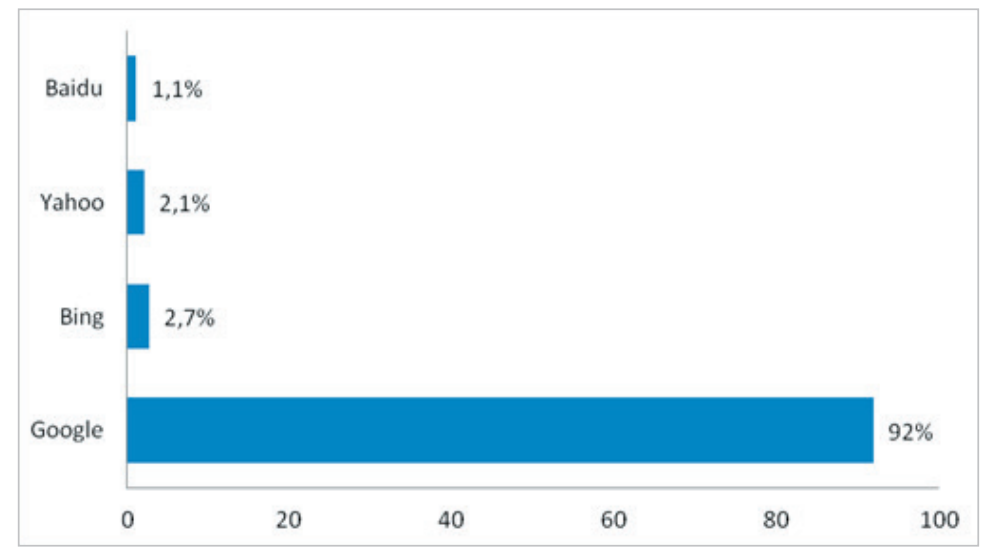

Gráfico 3. Cuota mundial del mercado de búsquedas en internet (2017). Fuente: Statcounter.

La pieza básica de la estrategia Google en el mercado de la movilidad fue crear una alianza con los principales fabricantes de móviles y desarrolladores de software entorno a la OHA (Open Handset Alliance) y lanzar su propio sistema operativo Android.

Al margen de las ventajas de Android como sistema operativo frente a sus competidores, Google adoptó la decisión de desarrollarlo sobre Linux que

"como software abierto permitió a los fabricantes de móviles añadir sus propias mejoras incluyendo capacidades tanto a nivel hardware como software" (Mahmoudi; Nadi, 2018, p. 1).

La disponibilidad gratuita de Android colocó en una disyuntiva a los fabricantes de móviles que o bien no poseían su propio sistema operativo o si lo tenían era demasiado básico como para convertirse en una plataforma de servicios. La adopción de Android por parte de los principales fabricantes de móviles y un gran número de marcas blancas de gran penetración en los mercados asiáticos tuvo al menos 3 consecuencias importantes:

- Los fabricantes que no adoptaron Android como SO principal vieron como en poco tiempo sus ventas disminuyeron. El caso más significativo fue el de Nokia que en apenas 5 años pasó de líder mundial de ventas en smartphones a ser adquirida por Microsoft en 2013 para terminar reposicionándose en el mercado de los equipos de red (Doz; Wilson, 2018).

- La irrupción de Android condujo a la irrelevancia en este sector a Microsoft, propietaria de Windows y cuya estrategia 
en el mercado móvil se basó en clonar su modelo de venta del SO a los fabricantes. El fracaso de esta estrategia de Microsoft propició finalmente su completa salida del mercado móvil tras la venta de Nokia en 2016 y su renuncia a seguir desarrollando Windows Phone en 2017, reorientando por completo su actividad hacia los servicios en la nube bajo la nueva dirección de Satya Nadella (Briggs; Kassner, 2017).

- La adopción mayoritaria de Android por parte de fabricantes y posteriormente de usuarios, no sólo supuso la irrupción de un competidor frente a Apple sino que propició una alternativa frente a su sistema operativo iOS, sólo disponible para dispositivos de Apple.

En este nuevo e inédito escenario, el argumento del ahorro de costes que suponía para los fabricantes disponer de un SO gratuito y $100 \%$ compatible con todas las aplicaciones de Google junto a la citada posibilidad de hacer versiones propias, convencieron a éstos en poco tiempo de la idoneidad de Android para sus nuevos modelos de smartphones.

El esfuerzo inversor de Google para desarrollar un Android cada vez más adaptado a los nuevos usos de los dispositivos móviles y ofrecido gratuitamente a los fabricantes se explica porque el objetivo era propiciar un mayor acceso a internet por medio de sus aplicaciones, lo que redunda en un mayor uso de su buscador y en más ingresos publicitarios (Bathia; Deep; Sachdeva, 2012).

Con Android como sistema operativo líder para smartphones (gráfico 4), Google dispuso de una nueva plataforma para el mercado de la publicidad online y a la vez se dotó de un sistema compatible para lograr una transición controlada hacia una internet mayoritariamente basada en dispositivos móviles.

La apertura en 2008 de su propia tienda de aplicaciones, inicialmente denominada Android Market a semejanza de la de Apple, completó el nuevo ecosistema de Google en el mercado de la movilidad en abierta competencia con el de Apple. Posteriormente, las diferentes versiones de Android para objetos conectados, coches, TV y wearables reflejaron la voluntad de Google de convertir Android en un SO universal que diera soporte a su plataforma de servicios en la nube. La estrategia de Google con Android es por lo tanto integral, convirtiéndose en el nuevo mediador de todos los mercados de contenido y servicios, mediante un liderazgo logrado en base al modelo de gratuidad, lo que favorece la rápida creación de economías de escala al eliminar barreras de entrada.

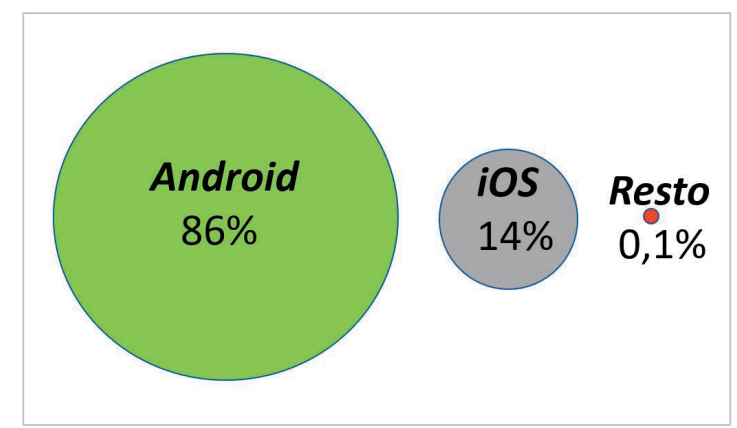

Grafico 4. Cuota de mercado de los sistemas operativos para móviles (2017). Fuente: Gartner, 2018.

Al igual que en el citado caso de Apple, la capacidad financiera de Alphabet y su positiva evolución en el mercado bursátil le permite realizar inversiones de riesgo en sectores ajenos a su actividad principal, mediante estrategias basadas en la competencia directa y de coste cero para el usuario. La diversificación de sus inversiones en sectores tan dispares como la conducción autónoma de vehículos (Waymo), el acceso y gestión de datos relativos a la salud (Verily) o el acceso a internet con su propia infraestructura de red (Google Fiber) aunque escasamente lucrativos hasta ahora (Alphabet, 2019) ofrecen a Alphabet una valiosa información sobre estos mercados y cómo la organización y acceso a estos datos podrían generar nuevos ingresos evitando una excesiva concentración del riesgo en torno al mercado publicitario.

\section{5G: convergencia tecnológica y evolución de sistemas}

Desde la perspectiva de la evolución de los sistemas de comunicación móviles, 5G se puede considerar como el sucesor de los actuales sistemas de cuarta generación (4G) definidos por el estándar IMT-Advanced (International Telecommunications Union - Radiocommunication, 2008) y cuyas primeras implementaciones comenzaron a verse en los principales mercados mundiales a comienzos de la segunda década de este siglo.

Aunque el desarrollo de un sistema de quinta generación que integrara distintos sistemas inalámbricos (wireless) preexistentes mediante una arquitectura abierta fue objeto de debate desde los comienzos mismos del 4G (Gohil; Modi; Patel, 2013) no fue hasta 2017 cuando la 3GPP definió las primeras normas para un sistema 5G NR (New Radio) no independiente (NSA), es decir que se apoyara sobre las redes 4G, permitiendo un periodo más largo de amortización de estas redes por las operadoras (Sutton, 2018).

En 2018 se publicaron las primeras normas 5 G como sistema independiente (SA), lo que significa que el 56 se podía considerar a partir de entonces técnicamente completado, abriéndose un periodo objetivamente mucho más largo en el cual las operadoras tienen que desarrollar la infraestructura de red, los fabricantes nuevos terminales de usuario y los gobiernos nacionales asignar nuevas frecuencias que en algunos casos desplazan servicios existentes, como es el caso de Europa con la televisión digital terrestre.

La estrategia de Google con Android es ser el nuevo mediador de todos los mercados de contenido y servicios, mediante un liderazgo logrado en base al modelo de gratuidad 
Técnicamente el $5 G$ supone una mejora respecto al $4 G$ en tres factores principales:

- velocidad de acceso (segmentos de subida y de bajada);

- latencia o tiempo que tarda una señal desde la estancia base al receptor;

- densidad de conexión o número de dispositivos que el sistema puede atender simultáneamente en condiciones óptimas en un espacio concreto y por unidad de tiempo (International Telecommunications Union - Radiocommunication, 2017) (gráfico 5).

Los tres factores son importantes para comprender no sólo la potencialidad tecnológica del 5G, sino sobre todo la necesidad de innovar en nuevos modelos de negocio ligados a esta tecnología (Lindgren, 2016) que permita a las operadoras recuperar la iniciativa en el mercado de los servicios sobre redes móviles o una mayor redistribución de los ingresos.

La velocidad derivada del ancho de banda empleado ha sido el factor principal para diferenciar las distintas generaciones de redes móviles avanzadas desde el $2 \mathrm{G}$ hasta el $4 \mathrm{G}$, pero también el argumento comercial más difundido por las operadoras para convencer a sus clientes de la necesidad de cambiar a un sistema superior. El 5G, bajo esta perspectiva, sigue esta tendencia al proporcionar velocidades hasta 20 veces mayores que su antecesor 4G, situando por primera a las redes móviles en el rango de los gigahertzs, algo que hasta ahora sólo se podía ofrecer mediante sistemas de fibra óptica.

En el inicio del despliegue del $5 \mathrm{G}$ las operadoras tienden a ofrecer una de las tres posibilidades que permite este sistema, el de mayor ancho de banda (eMBB, enhanced mobile broadband), ya que los otros dos, la comunicación masiva entre máquinas (mMTC, machine type communication) y latencia baja de alta fiabilidad (u-RLLC, ultra reliability and low latency communication), sólo se pueden ofrecer mediante un $5 \mathrm{G}$ completamente autónomo (SA) (Shafi et al., 2017).

Este incremento de la velocidad, a pesar de ser un parámetro importante para responder a los nuevos hábitos de consumo, se encuentra en poco tiempo con dos factores que actúan como barrera de entrada:

- la práctica inexistencia de una oferta de contenidos y servicios desarrollados específicamente para aprovechar los mayores anchos de banda del $5 \mathrm{G}$ [vídeo HDR (high dynamic range), AR (augmented reality), juegos, etc.].

- la existencia de sistemas físicos (cable, fibra) e inalámbricos (wifi, 4G, 3G) que cumplen suficientemente bien con la demanda actual y que además tienen una alta penetración en el mercado.

La latencia y densidad de conexión, aunque hasta ahora no han formado parte del argumento principal de venta de las redes de banda ancha móviles, constituyen el factor diferencial del $5 \mathrm{G}$ respecto a las generaciones anteriores. EI 4G actual ofrece unas latencias medias en torno a los 50/60 ms, suficiente no obstante para la transmisión de vídeo, mensajería multimedia y llamadas de voz de las aplicaciones más populares, pero insuficiente para servicios emergentes como la conducción autónoma, la cirugía remota y los juegos en realidad virtual (VR); por eso el $5 G$ en sus dos fases de despliegue reduce hasta $4 \mathrm{~ms}$ (en la modalidad eMBB) y $1 \mathrm{~ms}$ (en uRLLC) los tiempos medios de latencia (International Telecommunications Union - Radiocommunication, 2017).

La densidad de conexión del 5 G incrementa teóricamente este parámetro en un 100\% respecto al 4G elevándolo hasta un mínimo de un millón de dispositivos por $\mathrm{Km}^{2}$, lo que no solamente sirve para solventar problemas derivados de demandas puntuales como la alta concentración de dispositivos conectados en áreas reducidas como los eventos deportivos, sino sobre todo es el requerimiento básico para activar una loT masiva.

\section{Conclusiones: reinvención del mercado y redes 5G}

El liderazgo de Apple y Alphabet en los elementos clave del nuevo mercado de las comunicaciones sobre redes móviles ha convertido este sector en un duopolio, donde las operadoras a pesar de ser dueñas de las redes son incapaces de influir decisivamente en la marcha del mercado.

Este proceso de concentración, lejos de ser excepcional obedece a una de las tendencias largamente observadas en todos los sectores donde se emplean tecnologías de la información ya que 
“los beneficios de la innovación se concentran cada vez más en mercados donde el ganador se lleva todo [...] lo que conduce a una nueva categoría de empresas superestrellas" (European Commission, 2018, p. 7).

No obstante, el monopolio que representan en sus respectivos segmentos tanto Apple como Alphabet no responde a las características conocidas hasta ahora en este tipo de estructuras de mercado (Samuelson; Nordhaus, 2010), ya que ni existen altas barreras de entrada, ni son los únicos agentes del mercado, ni fijan uniteralmente el precio del producto y quizá lo más significativo, no se puede afirmar que ambas supongan un freno a la innovación en el sector.

Esta aparente contradicción se explica porque el mercado tecnológico actual se caracteriza por un alto grado de dinamismo. Hay que tener en cuenta que

"en un mundo estático, el monopolista es un simple recolector de rentas. Pero vivimos en un mundo dinámico: es posible inventar nuevas y mejores cosas. Los monopolistas creativos ofrecen a los clientes más opciones añadiendo nuevas categorías de abundancia al mundo" (Thiel; Masters, 2014, p. 29).

En el mercado de las redes móviles las operadoras hasta ahora no han sabido ofrecer a sus clientes servicios de alto valor añadido, en parte por las limitaciones impuestas por la neutralidad de red, pero fundamentalmente por su escasa comprensión del "carácter descentralizado" de la innovación en internet (Benkler, 2006).

El análisis de este mercado demuestra que la innovación fundamentalmente no se ha producido en la infraestructura que soporta la red, sino en la forma de acceder a ésta. La consecuencia es que las estrategias comerciales de Alphabet y Apple han influido directamente en el ritmo de inversión de las operadoras pero no al revés.

La transición de $2 \mathrm{G}$ a $3 \mathrm{G}$ sirve como ejemplo, ya que las operadoras ofrecieron éste como primer sistema que realmente se podía calificar de banda ancha a principios de este siglo y sin embargo, hasta la presentación del primer iPhone de Apple en el 2007 no se produjo un cambio mayoritario en los hábitos de acceso de los usuarios de la telefonía móvil.

Estas lecciones derivadas del análisis de la evolución de los sistemas móviles digitales de banda ancha deben ser tenidas en cuenta por las operadoras en su estrategia hacia el 5G. Sobre todo, en lo referente a que un nuevo sistema que ofrezca básicamente mayores velocidades de acceso no implica necesariamente mayores beneficios.

La reducción del coste por gigabyte que supondrá la adopción de las redes $5 \mathrm{G}$ permitirá a las operadoras ofrecer unas tarifas de acceso más competitivas que las actuales, reduciendo las barreras de entrada para los 700 millones de usuarios mundiales de móviles que todavía no han dado el salto a las redes de banda ancha (International Telecommunications Union, 2018b). El problema es que estos usuarios se localizan mayoritariamente en países donde el ingreso medio por usuario es mucho más bajo que en Norteamérica, Europa Occidental y Japón (GSMA Intelligence, 2018), de modo que, aunque se incremente el número de clientes, el margen de beneficio no aumentará proporcionalmente.

Las lecciones derivadas del análisis de la evolución de los sistemas móviles digitales de banda ancha deben ser tenidas en cuenta por las operadoras en su estrategia hacia el $5 G$

En segundo lugar, a corto plazo ni Alphabet ni Apple tienen tentaciones de entrar de lleno en el mercado de las telecomunicaciones creando sus propias redes. Algunas experiencias que no lograron alcanzar los objetivos esperados, como el proyecto Google Fiber en EUA (Levin; Downes, 2018), parecen haber desalentado de momento a ambos a entrar en un mercado donde el dominio de la tecnología no es condición suficiente para ofrecer un buen servicio a un coste más competitivo que las operadoras convencionales.

Sin embargo, alguna de las nuevas tácticas empleadas por Apple, como la sustitución de la tarjeta SIM física por una aplicación de software (eSIM) que permite al usuario elegir en cada momento a qué red conectarse, sí supone una amenaza directa frente al tradicional poder de las operadoras de ofrecer una tarjeta SIM que sólo permite la conexión a una red. La eSIM facilita técnicamente al usuario la portabilidad de su línea a otro operador que temporalmente ofrezca mayores ventajas. El resultado es que las operadoras tendrán que flexibilizar su oferta y responder con mayor rapidez a los cambios en la demanda para fidelizar a sus clientes, asumiendo además que éstos podrían optar por redes abiertas, como los accesos wifi públicos, para continuar sin interrupciones sus comunicaciones.

Frente a estas amenazas evidentes la transición hacia el 5 G puede suponer ventajas estratégicas para las operadoras:

- Los fabricantes de dispositivos móviles necesitan acelerar esta transición como un argumento para acortar el ciclo de renovación de los aparatos, en un mercado con evidentes signos de madurez e incrementos de ventas cada vez más planos (Gartner, 2018). Este hecho convierte a los fabricantes en aliados de las operadoras, no en la inversión en infraestructura, pero sí en la necesidad de incentivar que sus clientes se conviertan cuanto antes en usuarios de redes $5 G$ y aprovechar al máximo las capacidades de sus renovados equipos.

- La transición del 4G al 5G para las operadoras no significa únicamente la disponibilidad de mayores velocidades de acceso para los usuarios particulares, sino una mayor profundización de su oferta de servicios para la industria. Un ejemplo de esta nueva relación estratégica de las operadoras con el resto de sectores industriales es convertirse en el soporte principal de la internet de las cosas, lo que significa 
"conectar prácticamente cualquier objeto todo el tiempo y en cualquier lugar" (Zhang; Chen, 2016, p. 2),

pero también los procesos ligados a éstos dotándolos de un grado de inteligencia que les permita enviar y recibir datos que informen sobre su estado o nivel de uso, lo que llevaría a un incremento de la eficacia en áreas como el diseño de productos, el marketing y la atención al cliente.

En general, los productores de aparatos domésticos y sectores como la automoción tienden a analizar la internet de las cosas y el $5 \mathrm{G}$ como una oportunidad excepcional para que los clientes adquieran productos nuevos sin esperar al final de su ciclo de renovación, que en algunos sectores puede extenderse hasta una década.

Sin embargo, la debilidad de este análisis es que la simple conexión de un aparato a una red no le asegura un grado de inteligencia. Si la única ventaja de cambiar un producto por otro igual pero conectado es que ofrece información valiosa al fabricante es más que probable que el cliente final posponga la renovación de éste al no encontrar ventajas efectivas.

En este escenario las operadoras de redes $5 \mathrm{G}$ podrían implicarse con los fabricantes de cualquier sector en aspectos clave como el I+D, compatibilidad con los distintos sistemas, analítica de datos y comercialización.

Alphabet y Apple, como las dos empresas más lucrativas de la internet móvil, no van a dejar pasar la oportunidad de extender su influencia en el nuevo negocio de la internet de las cosas y las redes 5G. Su estrategia durante la primera etapa de internet sobre redes móviles ha consistido en situar sus respectivas plataformas como el nuevo estándar de conexión, la verdadera interfaz de relación con el usuario, lo que les permitió convertirse en el mediador privilegiado entre fabricantes de equipos, operadores de red y proveedores de servicios.

Pero si Apple y Alphabet tratan de imponer sus respectivas plataformas a los fabricantes como el estándar mayoritario de la internet de las cosas, pueden convertirse en un freno más que un catalizador, ya que cualquier guerra comercial entre fabricantes de dispositivos incompatibles con ambos sistemas más que incentivar la compra tendería a retrasarla (Shapiro; Varian, 1999).

A la vez, con unos dispositivos conectados compatibles con las principales plataformas se volvería a un escenario donde el valor añadido para el usuario estaría más cerca de los servicios en la nube que en el propio hardware. Esto transformaría a la mayoría de los fabricantes de cualquier producto en proveedores de servicios de las grandes plataformas, lo que en principio devalúa su posición en la cadena de valor.

Por último, el 5 G supondrá para las operadoras la oportunidad de dirigirse a nuevos mercados de nicho troceando su red (network slicing), para ofertar servicios específicos a usuarios corporativos, ya sea mediante distintos anchos de banda, tiempos de latencia, densidades de conexión, o incluso diferentes frecuencias, lo que supondrá además un ahorro de costes respecto a disponer de todas las funcionalidades. Desde esta perspectiva el $5 G$ tiende a configurarse como "una plataforma de orquestación holística" (Nencioni et al., 2018) donde los distintos recursos se distribuyen a lo largo de la red, desde las estaciones base a los propios equipos del usuario.

Las redes $5 \mathrm{G}$ ocasionarán un efecto llamada para la innovación de actores no presentes todavía en el mercado y que podrían aportar una interpretación radicalmente diferente a los servicios que conocemos

No obstante, acercarse a necesidades más específicas y puntuales de cada sector industrial va a requerir una mayor flexibilidad no sólo a nivel tecnológico sino de gestión, lo que supone el mayor reto para las operadoras. Una nueva estrategia de las operadoras dirigida a analizar las necesidades presentes y futuras cliente a cliente, olvidando la oferta en paquetes cerrados, requiere una transformación de la propia cultura corporativa de estas empresas acostumbradas a mercados masivos con escasa variación, lo que se corresponde con la definición más aceptada de transformación digital (Tabrizi et al., 2019).

La diversificación de la oferta de servicios de las operadoras hacia nuevos nichos de mercado de gran potencial de crecimiento y con una demanda menos elástica, supone además una oportunidad para expandirse e incrementar la competencia en servicios de alto valor añadido como las redes definidas por software (SDN), la computación en la nube (cloud computing) o la seguridad.

Finalmente, las redes $5 \mathrm{G}$ por su mayor velocidad, su capacidad para integrar distintos sistemas y su flexibilidad operativa para atender demandas muy específicas, van a suponer un nuevo efecto llamada para la innovación de actores no presentes todavía en el mercado y que podrían aportar una interpretación radicalmente diferente de la oferta de servicios que conocimos hasta ahora.

\section{Referencias}

Alphabet Inc. (2018). Form 10-K.

https://abc.xyz/investor/static/pdf/20171231_alphabet_10K.pdf

Alphabet Inc. (2019). Form 10-Q.

https://abc.xyz/investor/static/pdf/20190429_alphabet_10Q.pdf 
Andra, Usha (2017). "The latest mobile makeover: why and how unlimited plans are re-emerged". Cisco blog, March 22. https://blogs.cisco.com/sp/the-latest-mobile-makeover-why-and-how-unlimited-data-plans-have-re-emerged

Asimakopoulos, Grigorios; Whalley, Jason (2017). "Market leadership, technological progress and relative performance in the mobile telecommunications industry". Technological forecasting and social change, v. 123, pp. 57-67. https://doi.org/10.1016/j.techfore.2017.06.021

Atkinson, Robert; Ezell, Stephen (2012). Innovation economics: the race for global advantage. New Haven: Yale University Press. ISBN: 9780300205657

Ballon, Pieter (2009). "The platformisation of the European mobile industry". Communications \& strategies, v. 1, n. 75, pp. 15-34. https://ideas.repec.org/a/idt/journl/cs7501.html

Bathia, Ajay; Deep, Gagan; Sachdeva, Amit (2012). "Strategic analysis of search engine giant: a case study of Google Inc". International journal of computing and business research.

https://bit.ly/39As6uV

Benkler, Yochai (2006). The wealth of networks. How social production transforms markets and freedom. New Haven; London: Yale University Press. ISBN: 9780300125771

Bresnahan, Timothy; Greenstein, Shane (2014). "Mobile computing: The next platform rivalry". The American Economic Association, v. 104, n. 5, pp. 475-480.

http://www.jstor.org/stable/42920983

Briggs, Barry; Kassner, Eduardo (2017). Enterprise cloud strategy. Redmond: Microsoft Press. https://bit.ly/36qDsPQ

Case, Steve (2016). The third wave. An entrepreneur's vision of the future. New York: Simon \& Schuster. ISBN: 9781501132599

Christensen, Clayton M. (1997). The innovators dilemma. Boston: Harvard Business Review Press. ISBN: 9781633691780

Cisco (2017). The zettabyte era: trends and analysis.

https://bit.ly/39Gklna

Collins, Jim (1999). "Turning goals into results. The power of catalytic mechanisms". Harvard business review.

https://goo.gl/AsrNM1

Comisión Europea (2013). “Directrices de la Unión Europea para la aplicación de las normas sobre ayudas estatales al despliegue rápido de redes de banda ancha". Diario oficial de la Comisión, 26 enero.

https://eur-lex.europa.eu/LexUriServ/LexUriServ.do?uri=OJ:C:2013:025:0001:0026:ES:PDF

Corrocher, Nicoletta (2013). "The development of short message services: standard organizations as engines of innovations". Revue économique, v. 64, n. 1, pp. 149-163.

https://doi.org/10.3917/reco.641.0149

Curwen, Peter; Whalley, Jason (2004). Telecommunications strategy. Cases, theory and applications. New York: Routledge. ISBN: 9780415342384

Dediu, Horace (2010). "The iPhone is not superfluous, not easily copied, not revolutionary and not a premium product". Asymco, November 29.

https://goo.gl/vzzLXm

Dediu, Horace (2018). "The iOS economy updated". Asymco, January 8.

http://www.asymco.com/2018/01/08/the-ios-economy-updated

Doz, Yves; Wilson, Keeley (2018). Ringtone. Exploring the rise and fall of Nokia in mobile phones. Oxford: Oxford University Press. ISBN: 9780198777199

European Commission (2018). Science, research and innovation performance of the EU 2018.

https://ec.europa.eu/info/sites/info/files/rec-17-015-srip-report2018_mep-web-20180228.pdf

Evans, Benedict (2017). “Content isn't king”. Benedict Evans, July 16.

https://www.ben-evans.com/benedictevans/2017/7/13/content-isnt-king

Federal Communications Commission (FCC) (2018). 2018 Broadband deployment report. February 2.

https://www.fcc.gov/reports-research/reports/broadband-progress-reports/2018-broadband-deployment-report

Feijóo, Claudio; Maghiros, loannis; Abadie, Fabienne; Gómez-Barroso, José-Luis (2009). “Exploring a heterogeneous and fragmented digital ecosystem: mobile content". Telematics and informatics, v. 26, n. 3, pp. 282-292.

https://doi.org/10.1016/j.tele.2008.11.009

Forge, Simon; Horvitz, Robert; Lindmark, Sven; Bohlin, Erik; Blackman, Colin (2018). Fixed and mobile convergence in Europe. European Commission. 
https://horvitz.multiplace.org/IMIT-FMC-Final-Report-2018.pdf

Gartner (2018). "Gartner says worldwide sales of smartphones recorded first ever decline during the fourth quarter of 2017". Gartner, February $22^{\text {nd }}$.

https://cutt.ly/QruSePX

Gentzoglanis, Anastassios; Henten, Anders (2010). Regulation and the evolution of the global telecommunication industry. Cheltenham: Edward Elgar Publishing. ISBN: 9781848445888

Gohil, Asmitaba; Modi, Hardik; Patel, Shobhit K. (2013). “5G technology of mobile communication”. In: 2013 International conference on intelligence systems and signal processing ISSP.

https://doi.org/10.1109/ISSP.2013.6526920

GSMA (2018). The mobile economy 2018. GSM Association.

https://bit.ly/35oAf26

GSMA (2019). The mobile economy 2019. GSM Association.

https://www.gsmaintelligence.com/research/?file=b9a6e6202ee1d5f787cfebb95d3639c5\&download

GSMA Intelligence (2018). Definitive data and analysis for the mobile industry. GSM Association.

https://bit.ly/39AR7WB

Heinemann, Gerrit; Gaiser, Christian (2014). Social, local, mobile. The future of location-based services. New York: Springer. ISBN: 9783662439630

Holmes, Andrew (2008). Commoditization and the strategic response. London: Routledge. ISBN: 9780566087431

Ingraham, Nathan (2013). "iTunes store at 10: how Apple built a digital media juggernaut”. The verge, April 26.

https://bit.ly/2FmCEQo

Issarny, Valérie; Georgantas, Nikolaos; Hachem, Sara; Zarras, Apostolos; Vassiliadis, Panos; Autili, Marco; Gerosa, Marco-Aurelio; Hamida, Amira B. (2011). "Service-oriented middleware for the future of internet: state of the art and research directions". Journal of internet services and applications, v. 2, n. 1, pp 23-45.

https://doi.org/10.1007/s13174-011-0021-3

International Telecommunications Union (ITU) (2017). ICT data and statistics.

https://goo.gl/Vqddww

International Telecommunications Union (ITU) (2018a). Measuring the information society report 2018.

https://bit.ly/2ubaFR9

International Telecommunications Union (ITU) (2018b). The state of broadband 2018: broadband catalyzing sustainable development. Geneva: International Telecommunications Union. ISBN: 9789261264314

https://bit.ly/2NNNBAo

International Telecommunications Union - Radiocommunication (ITU-R) (2008). Requirements related to technical performance for IMT-Advanced radio interface(s). Report ITU-R M.2134.

https://www.itu.int/dms_pub/itu-r/opb/rep/R-REP-M.2134-2008-PDF-E.pdf

International Telecommunications Union - Radiocommunication (ITU-R) (2017). Minimum requirements related to technical performance for IMT-2020 radio interface(s). Report ITU-R M. 2410-0.

https://www.itu.int/dms_pub/itu-r/opb/rep/R-REP-M.2410-2017-PDF-E.pdf

Johnson, Katherine; Li, Yang; Phan, Hang; Singer, Jason; Trinh, Hoang (2012). "The innovative success that is Apple". Theses, dissertations and capstones, paper 418.

https://mds.marshall.edu/etd/418

Kenney, Martin; Pon, Bryan (2011). "Structuring the smartphone industry: Is the mobile internet OS platform the key?". Journal of industry, competition and trade, v. 11, n. 3.

https://link.springer.com/article/10.1007/s10842-011-0105-6

Kim, Ki Joon; Sundar, S. Shyam (2014). “Does screen size matter for smartphones? Utilitarian and hedonic effects on screen size on smartphone adoption". Cyberpsychology behavior and social networking, v. 17, n. 7, pp. 466-473. https://doi.org/10.1089/cyber.2013.0492

Klemperer, Paul (2002). "How (not) to run auctions: The European 3G telecom auctions". European economic review, n. 46. https://doi.org/10.2139/ssrn.297907

Laugesen, John; Yuan, Yufei (2010). "What factors contributed to the success of Apple's iPhone?”. In: 2010 Ninth international conference on mobile business and Ninth global mobility roundtable (ICMB-GMR), Athens, pp. 91-99.

https://doi.org/10.1109/ICMB-GMR.2010.63 
Levin, Blair; Downes, Larry (2018). "Why Google Fiber is high-speed internet's most successful failure”. Harvard business review, September 7.

https://hbr.org/2018/09/why-google-fiber-is-high-speed-internets-most-successful-failure

Lindgren, Peter (2016). "Multi business model innovation in a world of 5G: What will persuasive business models look like in a world of 5G?". Wireless personal communications, n. 88.

https://doi.org/10.1007/s11277-016-3243-7

Mahmoudi, Mehran; Nadi, Sarah (2018). "The Android updated problem: an empirical study". In: MSR 2018, 15 th International conference on mining software repositories, Gothenburg.

https://arxiv.org/pdf/1801.02716.pdf

Mölleryd, Bengt G.; Markendahl, Jan; Werding, Jan; Mäkitalo, Östen (2010). "Decoupling of revenues and traffic-Is there a revenue gap for mobile broadband?". In: $9^{\text {th }}$ Conference of telecommunication, media and internet, CTTE 2010. https://doi.org/10.1109/CTTE.2010.5557696

Montgomery, Johnna; Roscoe, Samuel (2013). "Owning the consumer. Getting to the core of the Apple business model". Acounting forum, v. 37, n. 4, pp. 290-299. https://doi.org/10.1016/j.accfor.2013.06.003

Naeem, Ibrahim (2016). Grounding the blue ocean strategy on technological innovations and network externalities. Yasar University Institute of Social Sciences, Master thesis.

https://bit.ly/2MW4hUy

Nencioni, Gianfranco; Garroppo, Rosario G.; González, Andrés J.; Helvik, Bjarne E.; Procissi, Gregorio (2018). “Orchestration and control in software-defined $5 \mathrm{G}$ networks: research challenges". Wireless communications and mobile computing, v. 2018, article ID 6923867.

https://doi.org/10.1155/2018/6923867

Rogers, Jim (2013). The death and life of the music industry in the digital age. New York: Bloomsbury. ISBN: 9781 623560010

Samuelson, Paul; Nordhaus, William (2010). Economics. New York: McGraw Hill. ISBN: 9780073511290

Sarwar, Muhammad; Soomro, Tarik R. (2013). "Impact of smartphone's in society". European journal of scientific research, v. 98, n. 2, pp 216-226.

https://pdfs.semanticscholar.org/2c28/0b6a690442a97a571e09b2404e2d21720db4.pdf

Schmidt, Eric; Rosenberg, Jonathan (2014). How Google works. New York: Grand Central Publishing. ISBN: 9781455582341

Shafi, Mansoor; Molisch, Andreas F.; Smith, Peter J.; Haustein, Thomas; Zhu, Peiying; De-Silva, Prasan; Tufvesson, Fredrik; Benjebbour, Anass; Wunder, Gerhard (2017). “5G: A tutorial overview of standards, trials, challenges, deployment and practice". IEE Journal on selected areas in communications, v. 35, n. 6.

https://ieeexplore.ieee.org/document/7894280

Shapiro, Carl; Varian, Hal R. (1999). "The art of standard wars". California management review, n. 41. https://es.scribd.com/document/68387635/The-Art-of-Standards-Wars

Sutton, Andy (2018). "5G network architecture". The ITP (Institute of Telecommunications Professionals) journal, v. 12, n. 1, pp. 9-15.

https://www.academia.edu/36284890/5G_Network_Architecture

Tabrizi, Behnam; Lam, Ed; Girard, Kirk; Irvin, Vernon (2019). "Digital transformation is not about technology". Harvard business review, March 13.

https://hbr.org/2019/03/digital-transformation-is-not-about-technology

Thiel, Peter; Masters, Blake (2014). Zero to one. Notes on startups or how to build the future. New York: Crown Business. ISBN: 9780804139304

Thorndike, Edward L. (1920). "A constant error in psychological ratings. Journal of applied psychology, v. 4, n. 1, pp. 25-29. https://doi.org/10.1037/h0071663

Varian, Hal (2003). "Economics of information technology". Mattioli Lecture Bocconi University http://people.ischool.berkeley.edu/ hal/Papers/structure.pdf

Zhang, Yin; Chen, Min (2016). Cloud based 5G wireless networks. Springer Briefs in computer science. ISBN: 9783319473437

Zittrain, Jonathan (2008). The future of internet and how to stop it. New Haven \& London: Yale University Press. ISBN: 9780300151244 